\title{
Space-time signal processing in MIMO antennas system with the Earth surface reflections
}

\author{
Igor Krychkov, Gennady Slukin, and Valery Chapursky* \\ Bauman Moscow State Technical University (BMSTU), 2-nd Baumanskaya str., 5, b. 1. \\ 105005 Moscow, Russia
}

\begin{abstract}
The generalized correlation integral for space-time signal processing at the output of a MIMO antenna system radio altimeter in the Earth's surface reflections presence is obtained. The results of calculation of one-dimensional cross-section of the generalized correlation integral at an elevation angle with a known target range and two-dimensional crosssection of the generalized correlation integral «horizontal range-height» for specific surface reflection coefficient are presented.
\end{abstract}

\section{Introduction}

Since the beginning of the 2000s, MIMO technology based on usage of antenna arrays with transmitting elements, which emit orthogonal signals used in information transmission systems, has found application in small-size radars creating [1,2]. Probe signal components scattered from the target can be separated at the outputs of transmitting elements according to their orthogonality. Similar solutions took place in the long-range radar RIAS [3], produced in 80-90s of the last century, when there was no MIMO technology in information transmission systems. Usage of the MIMO antenna systems leads to a significant reduction of the total number of elements in antenna array, simplifying the element feeding and microwave wiring of signals, which is important in small-sized radars development. Resolution performance of MIMO radar in case of monitoring targets in free space were analysed and well explored in numerous papers, for example in [1-3].

In this paper we enounce new theoretical results in the context of monitoring pin-point target in presence of earth or water surface reflections. In this paper we derived and quantitatively analyzed the expression for generalized correlation integral for space-time signal processing of orthogonal signals scattered by the target and its "antipode" [4], simulating earth surface reflections.

Some example of calculating the cross sections of the generalized correlation integral by elevation and "distance-height" coordinates accompanied by specific surface reflection coefficient for a radar altimeter with MIMO antenna system are given in this paper.

\section{Generalized correlation integral over spatial coordinates for MIMO radio altimeter}

To simplify the presentation of the theoretical analysis method in case of monitoring a target above the Earth surface in MIMO radar, we considered the case in which the earth

\footnotetext{
*Corresponding author: valch2008@yandex.ru
} 
surface is flat and the roughness coefficient $\dot{\rho}$ lies within the limit $0<|\dot{\rho}|<1$. As it is known, the definition of an «antipode», which allows to substitute the reflection of probe signal from the reflecting surface with scattering signals from both the target and the «antipode», is introduced for a target situated above a reflecting surface [4]. The main feature of the MIMO radar is that this fact is true for all of the pairs of receiving and transmitting elements, specifically for the orthogonal component of probe signals on propagation ways such as «the $n$-th transmitting element - target - the $k$-th receiving element and the $n$-th transmitting element - antipode - the $k$-th receiving element. Figure 1 illustrates the ray path geometry for the pair « $n$-th transmitting element» and « $k$-th receiving element» for the target $(T)$ and its antipode $(A)$.

In this paper we considered a MIMO antenna system consists of linear transmitting and receiving subarrays, when the radar operates in radar altimeter mode. This fact made it possible to approximately substitute each of the subarrays with its phase center, which has a wide radiation pattern in vertical plane, and to present the whole antenna system as a vertical linear MIMO antenna array which consists of $N_{t}$ transmitting and $N_{r}$ receiving phase centers with coordinate vectors $\mathbf{r}_{t n}$ and $\mathbf{r}_{r k}$. Figure 1a illustrates an example of the phase centers locations in such MIMO antenna system with $N_{t}=4$ and $N_{r}=8$. There is a concept of a virtual antenna array for MIMO antenna arrays [1,2], in accordance to which the coordinates of virtual centers are calculated as half-sums of the coordinates of all possible pairs of transmitting and receiving elements. Figure $1 \mathrm{~b}$ illustrates a virtual antenna array consisting of transceiver phase centers corresponding to linear MIMO antenna array composed of phase centers shown on figure 1a. The total number of virtual phase centers in that case can be calculated as $N_{t} N_{r}=32$, which significantly exceeds the number of real phase centers that equals $N_{t}+N_{r}=12$.

A characteristic feature of a MIMO radar in case of monitoring a target in the presence of surface reflections is that these reflections should be taken into account for all different propagation ways corresponding to all pairs «transmitting element - receiving element» separated in space. For each pair a specific composition of propagation ways is required for real target and its antipode, shown on figure 2 in case when the target is located on a vertical plane with coordinates $\left(0, y_{T}, z_{T}\right)$ and coordinates of phase centers of transmitting and receiving subarrays $z_{t n}$ and $z_{r k}$ on $z$ axis. This is directly related to the virtual MIMO antenna array shown on figure 1a.

Due to the fact that the antenna system is located on the plane $x O y$, coordinate vectors of phase centers of the $n$-th linear horizontal transmitting subarray and the $k$-th linear receiving subarray in a MIMO radar are equal $\mathbf{r}_{t n}=\left\|0,0, z_{t n}\right\|$ and $\mathbf{r}_{r k}=\left\|0,0, z_{r k}\right\|$ respectively. Transmitting phase centers emit chirp probe signals with mutually orthogonal components at frequencies $\omega_{n}$ with the step $\Delta \omega$ and complex envelope $\dot{U}(\mathrm{t})$ :

$$
\dot{s}_{n}(t)=\dot{U}(t) \exp \left(j \omega_{n} t\right), \quad \omega_{n}=\omega_{0}+(n-1) \cdot \Delta \omega, n=1, \ldots, N_{t} .
$$

The shape of the complex amplitude spectrum modulus $\left|G_{u}(\omega)\right|$ is close to rectangular [5] and the spectrums of orthogonal partial probe signals (1) practically do not overlap in case of chirp signals with a large time-frequency base and a frequency deviation equal to the frequency step $\Delta \omega$ in (1).

In the presence of surface reflections, pin-point target $\mathrm{T}$ and its antipode $\mathrm{A}$ are considered with coordinate vectors $\mathbf{R}=\|x, y, z\|$ and $\mathbf{R}^{\prime}=\|x, y,-z\|$, where $z$ is a height of the target, and $x, y$ are the horizontal plane coordinates.

In case of using broadband probe signals of the form (1) and, in particular, the chirp probe signals, the analysis of interference surface reflections has a certain peculiarity in 


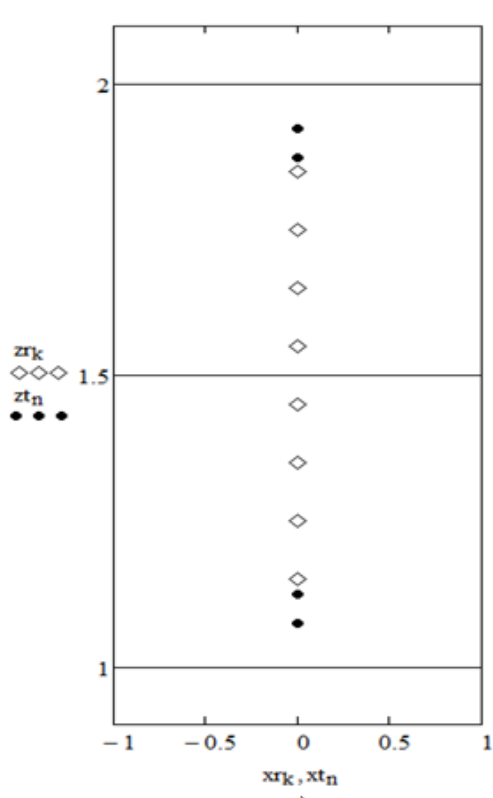

a)

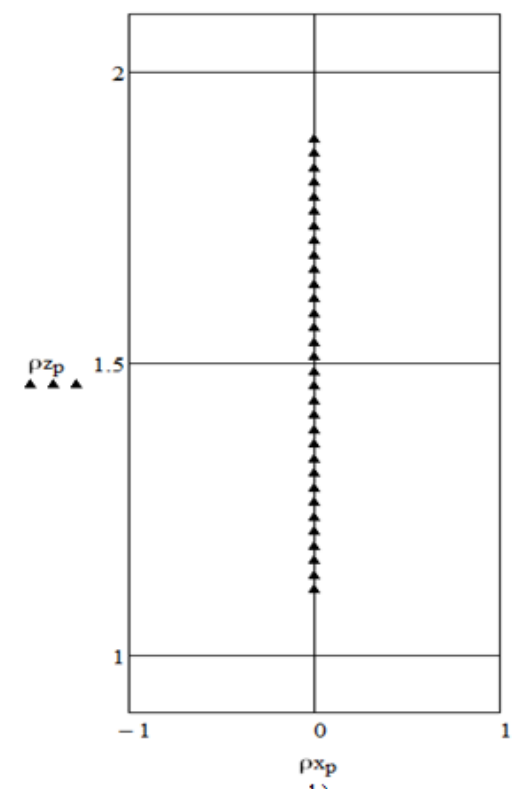

b)

Fig. 1. An example of the phase center locations in linear subarrays in MIMO antenna systems (a) and a virtual antenna array consisting of transceiver phase centers (b). The center of MIMO antennas system is located at the height of $1.5 \mathrm{~m}$; rhombuses - receiving phase centers; black ones transmitting phase centers; triangles - virtual transceiver phase centers. The axis coordinates on the figure are in meters.

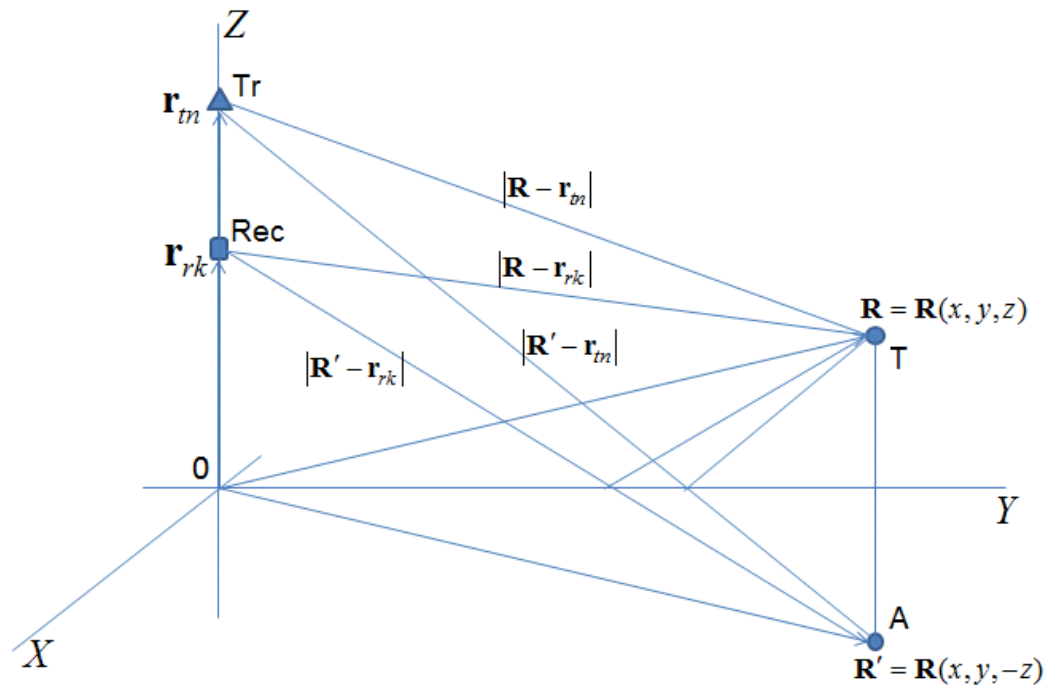

Fig. 2. Ray paths for a pair of phase centers of transmitting and receiving subarrays of MIMO radar, target $(\mathrm{T})$ and antipode $(\mathrm{A})$.

comparison with narrowband signals, caused by the high resolution of delays in broadband probe signals. During the analysis let us denote the propagation delays of the signals in following way: «phase center of the $n$-th transmitting subarray - target at the point with coordinates $\mathbf{R}$ » as $\tau_{n}=\tau_{n}(\mathbf{R})$; «phase center of the $n$-th transmitting subarray - antipode at the point with coordinates $\mathbf{R}^{\prime}$ » as $\tau_{n}^{\prime}=\tau_{n}\left(\mathbf{R}^{\prime}\right)$; «target at the point $\mathbf{R}$ - phase center of the 
$k$-th receiving subarray» as $\theta_{k}=\theta_{k}(\mathbf{R})$; «antipode at the point with coordinates $\mathbf{R}^{\prime}$ - phase center of the $k$-th receiving subarray» as $\theta_{k}^{\prime}=\theta_{k}\left(\mathbf{R}^{\prime}\right)$, where

$$
\tau_{n}(\mathbf{R})=c^{-1}\left|\mathbf{R}-\mathbf{r}_{t n}\right|, \theta_{k}(\mathbf{R})=c^{-1}\left|\mathbf{R}-\mathbf{r}_{r k}\right| .
$$

Taking into account the interference from the surface with a complex reflection coefficient $\rho$, the signal from the $n$-th transmitting subarray at the target with consideration of the shape of the probe signal (1) will be

$$
\dot{u}_{n}(t)=U_{0}\left[\dot{s}_{n}\left(t-\tau_{n}\right)+\dot{\rho} \dot{s}_{n}\left(t-\tau_{n}^{\prime}\right)\right],
$$

where $U_{0}$ is an amplitude conditioned by the propagation. The signal at the output of the $k$ th receiving subarray, corresponding to the reflected target signal, due to the $n$-th transmitting subarray, is equal to

$$
\dot{v}_{n k}(t)=U_{1}\left[\dot{u}_{n}\left(t-\theta_{k}\right)+\dot{\rho} \dot{u}_{n}\left(t-\theta_{k}^{\prime}\right)\right],
$$

where $U_{1}$ is an amplitude also conditioned by the propagation.

Substituting (3) into (4) and adding up over all transmitting subarrays, we obtain a signal corresponding to the output signal of the $k$-th receiving subarray for a pair of reflectors «target at the point with coordinates $\mathbf{R}$ - antipode at the point with coordinates $\mathbf{R}^{\prime} »$ :

$$
\dot{S}_{k}(t, x, y, z)=U_{0} U_{1} \sum_{n=0}^{N_{t}}\left\{\begin{array}{c}
\left.\dot{S}_{n}\left(t-\tau_{n k}^{(0)}(x, y, z)\right)+\dot{\rho}\left[\dot{s}_{n}\left(t-\tau_{n k}^{(1)}(x, y, z)\right)+\dot{s}_{n}\left(t-\tau_{n k}^{(2)}(x, y, z)\right)\right]+\right\} \\
+\dot{\rho}^{2} \dot{s}_{n}\left(t-\tau_{n k}^{(3)}(x, y, z)\right)
\end{array}\right\} .
$$

Here we introduced and denoted sums of delays $\tau_{n}+\theta_{k}$ in a form of (2) for different propagation ways from phase centers of subarrays for the target and the antipode with vectors depending on the Cartesian coordinates $x, y, z$, respectively considering the fact that $\mathbf{R}=\mathbf{R}(x, y, z)$ and $\mathbf{R}^{\prime}=\mathbf{R}(x, y,-z):$

$$
\begin{aligned}
& \tau_{n k}^{(0)}(x, y, z)=c^{-1}\left\{\left|\mathbf{R}(x, y, z)-\mathbf{r}_{t n}\right|+\left|\mathbf{R}(x, y, z)-\mathbf{r}_{r k}\right|\right\}, \\
& \tau_{n k}^{(1)}(x, y, z)=c^{-1}\left\{\left|\mathbf{R}(x, y, z)-\mathbf{r}_{t n}\right|+\left|\mathbf{R}(x, y,-z)-\mathbf{r}_{r k}\right|\right\}, \\
& \tau_{n k}^{(2)}(x, y, z)=c^{-1}\left\{\left|\mathbf{R}(x, y,-z)-\mathbf{r}_{t n}\right|+\left|\mathbf{R}(x, y, z)-\mathbf{r}_{r k}\right|\right\}, \\
& \tau_{n k}^{(3)}(x, y, z)=c^{-1}\left\{\left|\mathbf{R}(x, y,-z)-\mathbf{r}_{r k}\right|+\left|\mathbf{R}(x, y,-z)-\mathbf{r}_{t n}\right|\right\} .
\end{aligned}
$$

As a reference signal, let us choose a signal corresponding to the reference point in space with a vector of coordinates $\mathbf{R}_{0}=\mathbf{R}\left(x_{0}, y_{0}, z_{0}\right)$. Taking (1) into consideration, it equals:

$$
\dot{S}_{k}\left(t, x_{0}, y_{0}, z_{0}\right)=\sum_{n=0}^{N_{t}} \dot{s}_{n}\left(t-\tau_{n k}\left(\mathbf{R}\left(x_{0}, y_{0}, z_{0}\right)\right)\right) .
$$

Then for generalized correlation integral:

$$
\dot{Q}\left(x_{0}, y_{0}, z_{0}, x, y, z\right)=\sum_{k=0}^{N_{r}} \int_{t} \dot{S}_{k}(t, x, y, z) \dot{S}_{k}^{*}\left(t, x_{0}, y_{0}, z_{0}\right) d t,
$$

where symbol $«{ }^{*} »$ means complex conjugate value. 
The conversion of the generalized correlation integral (1) into a spectral form according to the technique [6] and the consideration of a nearly rectangular form of the specters of partial probe phase centers signals, that are orthogonal to one another, for each of subarrays allowed to obtain the following expression in the presence of surface reflections with a complex reflection coefficient $\dot{\rho}$ for generalized correlation integral in a MIMO radio altimeter with accuracy up to a constant factor:

$$
\left.\left.\dot{Q}\left(\mathbf{r}, \mathbf{r}_{0}\right)=\sum_{k=0}^{N_{r}} \sum_{n=0}^{N_{t}}\left\{q_{n k}^{(0)}\left(\mathbf{r}, \mathbf{r}_{0}\right)\right)+\dot{\rho} \cdot\left[q_{n k}^{(1)}\left(\mathbf{r}, \mathbf{r}_{0}\right)+q_{n k}^{(2)}\left(\mathbf{r}, \mathbf{r}_{0}\right)\right]+\dot{\rho}^{2} \cdot q_{n k}^{(3)}\left(\mathbf{r}, \mathbf{r}_{0}\right)\right)\right\} .
$$

where $q_{n k}^{(p)}, p=0,1,2,3$, can be calculated as following:

$$
\left.q_{n k}^{(p)}\left(\mathbf{r}, \mathbf{r}_{0}\right)\right)=\operatorname{sinc}\left[\frac{\Delta \omega}{2} \Delta \tau_{n k}^{(p)}\left(\mathbf{r}, \mathbf{r}_{0}\right)\right] \exp \left\{j \omega_{n} \Delta \tau_{n k}^{(p)}\left(\mathbf{r}, \mathbf{r}_{0}\right)\right\}, \Delta \tau_{n k}^{(p)}\left(\mathbf{r}, \mathbf{r}_{0}\right)=\tau_{n k}^{(0)}\left(\mathbf{r}_{0}\right)-\tau_{n k}^{(p)}(\mathbf{r})
$$

where $\mathbf{r}_{0}=\left\|x_{0}, y_{0}, z_{0}\right\|$ is a reference point vector, $\tau_{n k}^{(p)}\left(\mathbf{r}_{0}\right)=\tau_{n k}^{(p)}\left(x_{0}, y_{0}, z_{0}\right)$ and $\tau_{n k}^{(p)}(\mathbf{r})=\tau_{n k}^{(p)}(x, y, z)$ are determined using formulas (6)-(9).

The generalized correlation integral (12) can be denoted as functions of vectors $\mathbf{r}=\|r, \beta, \varepsilon\|$ and $\mathbf{r}_{0}=\left\|r_{0}, \beta_{0}, \varepsilon_{0}\right\|$ by using spherical coordinates.

Figure 3 illustrates examples of two-dimensional cross-sections of the generalized correlation integral for a vertical MIMO antenna array consisting of horizontal subarrays that simulates the operation of a short-range S-band MIMO radar in radio altimeter mode. The antenna array consists of 8 receiving and 4 transmitting phase center subarrays. Two pairs of transmitting elements are located both at the top and bottom of the antenna array receiving module to provide a distance between the virtual antenna array elements equal $0,25 \lambda[1,2]$. Full size of the antenna array equals 0,8 meter. Full bandwidth equals $20 \mathrm{MHz}$ with a frequency step and chirp probe signal bandwidth of each phase center equal $5 \mathrm{MHz}$. Surface reflection coefficient was assumed equal $|\dot{\rho}|=0,5$.

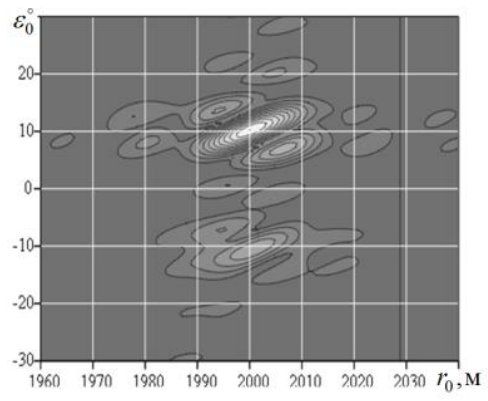

a)

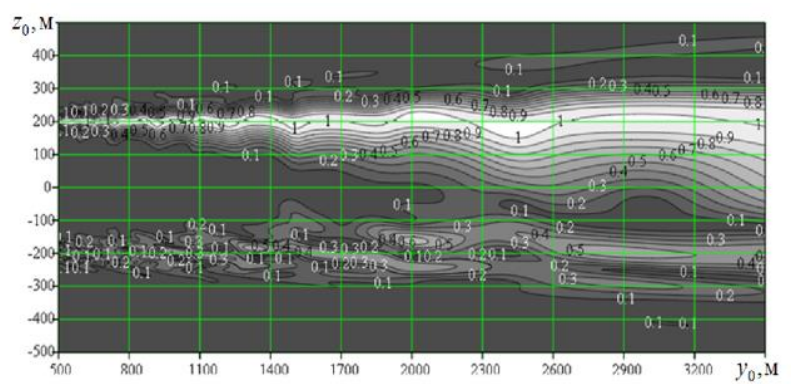

b)

Fig. 3. Two-dimensional cross sections of the normalized module of the generalized correlation integral set in: a) polar coordinates $\left(r_{0}, \varepsilon_{0}\right)$ with following conditions: $r=2000$ meters, $\varepsilon=10^{\circ}$, $\beta=0$; b) Cartesian coordinates $\left(x_{0}, y_{0}\right)$ with following conditions: $x=x_{0}=0$ and $z=200$ meters.

On each figure ( $3 \mathrm{a}$ and $3 \mathrm{~b}$ ), the peaks are visible corresponding to the elevation angle and the height of the real target and the antipode, that allow us to estimate the resolution and systematic errors of elevation angle or target height estimation with consideration of the influence of artifacts caused by reflections from the surface. One can see that with the increase of horizontal distance to the target, variations in the maximum of module of the generalized correlation integral appear, allowing to estimate the systematic error in measurement of the target height, due to the influence of the antipode. Similar two- 
dimensional cross-sections of the module of the generalized correlation integral at different reflection coefficients allow to qualitatively and quantitatively estimate the impact of reflections, for example, from the sea surface at different degrees of sea disturbance.

\section{Conclusion}

In this paper we propose a mathematical method based on the calculation of multidimensional generalized correlation integral of the space-time signal processing in short-range MIMO radar operating as a radio altimeter capable of monitoring targets with reflections of the transmitting elements' signals from the earth's surface in the background. In this paper quantitative results are presented for altitude measurement in the S-band MIMO radar with specific parameters of the antenna system and probe signal.

The proposed theoretical method allows generalization in case of a MIMO antennas system with arbitrary subarrays, as well as other configurations of MIMO antennas systems, which is important in the process of predesign development of technical appearance of a MIMO antennas system for low-altitude MIMO radars. This technique can also be used for comparing MIMO radars with different traditional radars.

\section{References}

1. J. Klare, O. Saalmann, MIRA-CLE X: A new Imaging MIMO-Radar for Multi-Purpose Applications. Proceedings of the 7th European Radar Conference, Paris, France., P. 129-132 (2010)

2. O. Biallawons, J. Klare, O. Saalmann, Technical realization of the MIMO radar MIRA CLE Ka. Proceedings of the 10th European Radar Conference, Nuremberg, Germany. P. 21-24 (2013)

3. J. Dorey, Y. Blanchard, F. Christophe, Le projet RIAS, un approche nouvelle du radar de surveilance aerienne. Colloque International dur le radar. Versailles (1984)

4. D. Barton. Radiolokacionnoe soprovojdenie tselei pri malih uglah mesta. TIIER, v. 42, No 6, pp. 37-61 (1974)

5. I. S. Gonorovsky, Radiotehnicheskie tsepi i signali, P.1. "Sov.Radio". (Moscow, 1967)

6. V. Iu. Loskutov, G. P. Slukin, V. V Capursky. Spektralnii metod obrabotki signalov v mnogochactotnih prostranstvenno mnogokanalnih RLS. Padiotehnika, No 11, pp. 3949 (2011) 